\title{
PROPER HOMOTOPY CLASSIFICATION OF GRAPHS
}

\author{
R. AYAlA, E. DOMINGUEZ, A. MARQUEZ AND A. QUINTERO
}

\begin{abstract}
This work presents a classification of the proper homotopy types of locally finite 1-dimensional CWcomplexes.
\end{abstract}

\section{Introduction}

A proper map (p-map) is a continuous map $f: X \rightarrow Y$ such that $f^{-1}(K)$ is compact for any compact $K$. Proper homotopy (p-homotopy), proper homotopy equivalence, etc., are defined in the natural way. A graph is the underlying space of a connected locally finite 1-dimensional CW-complex. We consider on the half-line $\mathbb{R}^{+}$the natural $\mathrm{CW}$-structure whose 0 -cells are the set of positive integers. Any cellular embedding $f: \mathbb{R}^{+} \rightarrow G$ is called an infinite branch of the graph $G$. The vertex $f(0)$ is called the root vertex of the branch.

It is a well-known fact that any compact graph has the same ordinary homotopy type as a wedge of finitely many copies of the 1 -sphere $S^{1}$. We call that number the genus of the graph. So the genus classifies the ordinary homotopy type of compact graphs and actually gives us an equivalence with the category of finitely generated free groups. Nevertheless, there are easy examples of non-compact graphs with the same ordinary homotopy type but different p-homotopy types. The aim of this work is to state the corresponding proper homotopy classification of non-compact graphs. This problem was posed to us by Professor H. J. Baues as the starting point for the study of proper homotopy from a combinatorial point of view.

As in the case of open surfaces (see [6]), the notion of Freudenthal end is the main tool used for obtaining such a classification. A Freudenthal end of a space $X$ is an element of the inverse limit $\mathscr{F}(X)=\varliminf_{\lim } \pi_{0}(X-K)$, where $K$ ranges over the family of compact sets of $X$ and $\pi_{0}(X-K)$ stands for the set of connected components. When $X$ is a $T_{2}$-locally compact $\sigma$-compact space, we can use a countable sequence $K_{1} \subset K_{2}$ $\subset$... of compact subsets to obtain $\mathscr{F}(X)$. The topology of $X$ can be enlarged to a topology on $\tilde{X}=X \cup \mathscr{F}(X)$ in such a way that $\mathscr{F}(X)$ turns out to be homeomorphic to a closed set of the Cantor set (see [3] and [1]), as follows. The topology on $\tilde{X}$ is generated by the topology of $X$ and the sets $\tilde{U}=U \cup U^{*}$, where $U \in \pi_{0}\left(X-K_{n}\right)$ for some $n$ and $U^{*}$ is the set of ends given by the sequences $\left\{U_{n}\right\} \in \lim \pi_{0}\left(X-K_{n}\right)$ such that there is a positive integer $n_{0}$ with $U_{n_{0}} \subset U$.

Another useful proper invariant is the notion of proper end. A proper end is a $\mathrm{p}$ homotopy class of p-maps $f: \mathbb{R}^{+} \rightarrow X$. The set of proper ends of $X$ is denoted by $F(X)$ and there is a map $\theta$ from $F(X)$ onto $\mathscr{F}(X)$.

Received 11 September 1989; revised 8 December 1989.

1980 Mathematics Subject Classification 05CXX, 54C10, 55P15.

Bull. London Math. Soc. 22 (1990) 417-421 
A Freudenthal end $\alpha$ is said to be stable if $\theta^{-1}(\alpha)$ is just one point. Otherwise, $\alpha$ is non-stable or unstable. We denote the set of unstable ends of $X$ by $\mathscr{F}^{\text {ns }}(X)$, which turns to be a closed subset of $\mathscr{F}(X)$. In order to know whether a Freudenthal end $\alpha$ is stable or not, we can use the bijection $\lim ^{1} \pi_{1}\left(X-K_{j, * j}\right) \cong \theta^{-1}(\alpha)$ (see [5]), where ${ }^{*} j$ is a point in the component of $X-K_{j}$ belonging to $\alpha$ (see [1] for more details).

It is easy to check that any p-map $f: X \rightarrow Y$ induces a continuous map $f_{*}:\left(\mathscr{F}(X), \mathscr{F}^{\text {ns }}(X)\right) \rightarrow\left(\mathscr{F}(X), \mathscr{F}^{\text {ns }}(Y)\right)$ such that if $f$ is a p-homotopy equivalence, $f_{*}$ is a homeomorphism. Notice that there exist p-maps $f$ which are not p-homotopy equivalences but their induced $f_{*}$ are homeomorphisms.

If we embed Cantor's set in $[0,1] \times\{0\} \subset \mathbb{R}^{2}$, it is a well-known result that there is a binary tree, called the Cantor tree, embedded in $\mathbb{R}^{2}$ in such a way that the following conditions hold. (1) The number of vertices on the $i$ th level is $2^{i}$. (2) The space of boundary points of the embedded tree is Cantor's set. (3) There is a natural 1-1 correspondence between the points of the Cantor set and the set of infinite branches of the tree starting from the root vertex.

\section{Characteristic pair of a graph}

DEFINITION 1.1. A graph is $\infty$-stable or stable at infinity if any Freudenthal end of $X$ is stable. A graph is totally unstable if any end of $X$ is unstable.

The following results are easy to prove.

Proposition 1.2. (a) The stable ends and the unstable ends are p-homotopy invariants.

(b) A graph is $\infty$-stable if and only if its fundamental group is finitely generated.

(c) Any non-compact graph $G \neq \mathbb{R}^{+}$is p-homotopy equivalent to a graph with no endvertices.

DEFINITION 1.3. The genus of an $\infty$-stable graph $G$ is the rank of the fundamental group $\pi_{1}(G)$. The characteristic pair of an $\infty$-stable graph is $(\mathscr{F}, g)$, where $\mathscr{F}$ is the space of Freudenthal ends of $G$ and $g$ is the genus of $G$. The characteristic pair of a non- $\infty$-stable graph is $\left(\mathscr{F}, \mathscr{F}^{\text {ns }}\right)$.

Two characteristic pairs $\left(\mathscr{F}_{1}, g_{1}\right)$ and $\left(\mathscr{F}_{2}, g_{2}\right)$ are isomorphic if $g_{1}=g_{2}$ and $\mathscr{F}_{1}$ and $\mathscr{F}_{2}$ are homeomorphic. Two characteristic pairs $\left(\mathscr{F}_{1}, \mathscr{F}_{1}^{\mathrm{ns}}\right)$ and $\left(\mathscr{F}_{2}, \mathscr{F}_{2}^{\mathrm{ns}}\right)$ are isomorphic if there exists a homeomorphism of pairs $h:\left(\mathscr{F}_{1}, \mathscr{F}_{1}^{\text {ns }}\right) \rightarrow\left(\mathscr{F}_{2}, \mathscr{F}_{2}^{\text {ns }}\right)$.

Proposition 1.4. (a) Given a pair $(\mathscr{F}, g)$, where $\mathscr{F}$ is a closed subset of the Cantor set and $g$ is a natural number, there exists an $\infty$-stable graph whose characteristic pair is $(\mathscr{F}, g)$.

(b) Given a pair of closed subsets $\left(\mathscr{F}, \mathscr{F}^{\prime}\right)$ of the Cantor set there exists a graph whose characteristic pair is the given one.

Proof. Given a pair $(\mathscr{F}, g)$, we may consider in a natural way the Cantor subtree defined by the subspace $\mathscr{F}$, and then we glue a wedge of $g$ copies of 1 -spheres at the root vertex. We obtain in this way an $\infty$-stable graph whose characteristic pair is $(\mathscr{F}, g)$. A graph associated to $\left(\mathscr{F}, \mathscr{F}^{\prime}\right)$ is obtained by gluing exactly one 1 -sphere at each vertex belonging to an infinite branch determined by a point of $\mathscr{F}^{\prime}$. 
Definition 1.5. The graphs given in Proposition 1.4 are called canonical graphs. An unstable branch of a canonical graph is an infinite branch where there is an 1 -sphere attached at each vertex.

\section{Proper classification of graphs}

From Proposition 1.2(c) we shall deal only with graphs with no end-vertices. Given a non-compact connected graph $G$ with no end-vertices, we set up a sequence of compact connected graphs $K_{1} \subset K_{2} \subset \ldots$ with $\bigcup K_{1}=G$ and a spanning tree $T \subset G$, as follows.

Let $K_{1}$ be any connected compact subgraph of $G$. Let $K_{2}^{*}$ be the extension of $K_{1}$ by adding all the incident edges to $K_{1}$. Let $\left\{D_{j}^{1}\right\}$ be the connected components of $G-K_{1}$. The vertices in $\left(K_{2}^{*}-K_{1}\right) \cap D_{j}^{1}$ are joined by a finite tree $T_{j}^{1} \subset D_{j}^{1}$. We define $K_{2}$ by adding to $K_{2}^{\prime}=K_{2}^{*} \cup\left\{\bigcup_{j} T_{j}^{1}\right\}$ all the edges with both vertices in $K_{2}^{\prime}$. We iterate that process to obtain the above sequence. Notice that if $D_{j}^{n}$ is a connected component of $G-K_{n}$, we have that $\mathrm{cl}\left(K_{n}-K_{n-1}\right) \cap \mathrm{cl}\left(D_{j}^{n}\right)$ is a compact connected graph $L_{j}^{n}$ joined to $K_{n-1}$ with a set of edges $A_{j}^{n}$.

We now choose a spanning tree $T \subset G$ as follows. We take a maximal tree in $K_{1}$ and all the trees $T_{j}^{n}(n \geqslant 1)$. We join them with edges of $A_{j}^{n}$. Notice that those paths in $T$ going between two vertices of $K_{n}-K_{n-2}$ miss the subgraph $K_{n-2}$.

Proposition 2.1. Given $G$ and $T$ as above, there is a graph $G^{\prime}$ p-homotopically equivalent to $G$ with the same tree $T$ and such that all the cycles of $G^{\prime}$ are loops.

Proof. Given an edge $e \in G-T$ which determines a cycle of $G$, let $v_{e}$ and $w_{e}$ be the vertices of $e$ and $L_{e}$ be the unique path in $T$ running from $v_{e}$ to $w_{e}$. We subdivide $e$ by adding a new vertex $c_{e}$ and we attach a new edge $\gamma_{e}$ with vertices $v_{e}$ and $c_{e}$ to $G$. We can now glue a 2-disk $D_{e}^{2}$ identifying $\partial D_{e}$ with $L_{e} \cup \overline{c_{e} w_{e}} \cup \gamma_{e}$, where $\overline{c_{e} w_{e}}$ is the 1-cell defined by $c_{e}$ and $w_{e}$. After doing that for each vertex $e$ which does not belong to $T$ (notice that all those gluings are locally finite by the construction of $T$ ), we obtain a 2-dimensional $\mathrm{CW}$-complex $\bar{G}$ p-homotopically equivalent to $G$ relatively to $T$. Performing now the obvious collapses, we obtain a graph $G^{\prime}$ p-homotopically equivalent to $G$ and such that all the cycles are loops.

Proposition 2.2. Any graph $G$ is p-homotopically equivalent to a canonical graph.

Proof. By Proposition 2.1 we may assume that $G$ is a directed tree $T$ with some loops attached at the vertices. By an obvious p-homotopy equivalence we may consider that the number of incident edges from a vertex is 1 or 2 and the number of incident edges to a vertex is 1 (except the first vertex, which has no incident edge to it). Therefore the tree of $G$ is now a subtree of the Cantor tree.

If $G$ is $\infty$-stable, after a suitable p-homotopy equivalence we may regard all the loops at the root vertex. Otherwise we can argue in the same way for each maximal stable infinite branch whose root vertex belongs to an unstable branch. Only the problem of distributing the loops remains. We now take any vertex $v$ with $n>1$ loops and subdivide any incident edge from $v$ adding $(n-1)$ new vertices. We may remove $(n-1)$ loops from $v$ and put each of them at one new vertex. This completes the proof. 
Lemma 2.3. Let $L$ be a subtree of the Cantor tree. Given a finite disjoint open covering $\left\{V_{1}, V_{2}, \ldots, V_{n}\right\}$ of $\mathscr{F}(L)$, we can choose a compact subtree $L_{0} \subset L$ such that each set $U^{*}$ of ends defined by a component $U \subset L-L_{0}$ is included in some $V_{i}$.

Proof. We have that each $V_{i}$ is closed in $\mathscr{F}(L)$ and so compact. Since the family of sets $U^{*}$, where $U$ is a component of $L-K, K$ running over the compact sets of $L$, is an open basis for the topology of $\mathscr{F}(L)$, we can cover each $V_{i}$ with only finitely many $U^{*}$ and then we can easily choose the tree $L_{0}$ we are looking for.

Proposition 2.4. Let $G$ and $G^{\prime}$ be two canonical graphs with trees $L$ and $L^{\prime}$. Then any continuous map $h:\left(\mathscr{F}(G), \mathscr{F}\left(G^{\text {ns }}\right)\right) \rightarrow\left(\mathscr{F}\left(G^{\prime}\right), \mathscr{F}\left(G^{\prime \text { ns }}\right)\right)$ induces a p-map $h^{\prime}:\left(L, L^{\mathrm{ns}}\right) \rightarrow\left(L^{\prime}, L^{\prime \mathrm{ns}}\right)$ unique up to p-homotopy of pairs such that $h_{*}^{\prime}=h \mid \mathscr{F}(L)$.

Proof. We take an increasing sequence of compact subtrees $L_{1}^{\prime} \subset L_{2}^{\prime} \subset \ldots$ of $L^{\prime}$. Let $n_{1}$ be the number of connected components of $L^{\prime}-L_{1}^{\prime}$ and $v_{j}^{\prime}$ be the first vertex in the component $V_{j}^{\prime}\left(1 \leqslant j \leqslant n_{1}\right)$. Applying the above lemma to

$$
\left\{h^{-1} V_{1}^{\prime *}, h^{-1} V_{2}^{\prime *}, \ldots, h^{-1} V_{n_{1}}^{\prime *}\right\}
$$

we may choose a finite subtree $L_{1}$ of $L$ such that the ends defined by each component $V_{k}$ of $L-L_{1}$ are included in some $h^{-1} V_{j(k)}^{\prime *}$. Let $v_{k}$ be the first vertex in $V_{k}$. We define $h^{\prime}\left(v_{k}\right)=v_{f(k)}^{\prime}$. All the vertices in $L_{1}$ are mapped to the root vertex $v_{0}^{\prime}$ of $L^{\prime}$. Now we take $L^{\prime}-L_{1}^{\prime}$ and we argue replacing $L^{\prime}$ and $L$ by the maximal trees in each $V_{1}$ and the maximal tree in each $V_{k}$. So we may choose a finite subtree $L_{2} \subset L$ such that the set of ends defined by each component of $L-L_{2}$ is mapped by $h$ in the set of ends defined by some component of $L^{\prime}-L_{2}^{\prime}$. We define $h^{\prime}$ as above for the first vertices and $h^{\prime}(v)$ $=v_{\jmath(k)}^{\prime}$ if $v$ is a vertex in $L_{2}-L_{1}$ and $V_{k}$ is the component of $v$ in $L-L_{1}$. We may iterate this procedure and define inductively a p-map $h^{\prime}: L \rightarrow L^{\prime}$ such that $h_{*}^{\prime}=h \mid \mathscr{F}(L)$. Notice that $h^{\prime}$ actually maps $L^{\text {ns }}$ into $L^{\prime \text { ns }}$.

Let $\bar{h}$ be another p-map with $\bar{h}_{*}=h$. By using a proper simplicial approximation [2], we may assume that $\bar{h}$ maps vertices into vertices and $\bar{h}\left(v_{0}\right)=h^{\prime}\left(v_{0}\right)$, where $v_{0}$ is the root vertex of $L$. From the properness of $\bar{h}$ we can choose increasing sequences $K_{1} \subset K_{2} \subset \ldots \subset L$ and $L_{1}^{\prime} \subset L_{2}^{\prime} \subset \ldots \subset L^{\prime}$ of compact subtrees such that any component of $L-K_{j}$ is mapped by $\bar{h}$ into a component of $L^{\prime}-L_{j}^{\prime}$. We can easily define a sequence $L_{n_{1}} \subset K_{n_{1}} \subset L_{n_{2}} \subset K_{n_{2}} \subset \ldots$, where $L_{n_{j}}$ is given in the definition of $h^{\prime}$.

Let $A_{j}$ be the set of first vertices of components of $L-K_{n_{j}}$. As $h_{*}^{\prime}=\bar{h}_{*}=h$, we claim that $h^{\prime}(v)$ and $\bar{h}(v)$ are in the same component of $L^{\prime}-L_{n_{j}}^{\prime}$ if $v \in A_{j}$. Indeed, if $\bar{h}(v) \in C_{1}^{\prime}$ and $h^{\prime}(v) \in C_{2}^{\prime}$ then $h^{-1} C_{1}^{\prime *}$ and $h^{-1} C_{2}^{\prime *}$ are disjoint open sets in $\mathscr{F}(L)$, and taking an end $\alpha$ starting from $v$ we obtain that $h(\alpha) \in C_{1}^{\prime *} \cap C_{2}^{\prime *}$. So we may take a path $\varepsilon_{v}$ in $L^{\prime}-L_{n,}^{\prime}$ going from $\bar{h}(v)$ to $h^{\prime}(v)$. In this way we can define the p-map

$$
H_{0}: L \times\{0,1\} \cup\left\{v \times I ; v \in \bigcup_{j=1}^{\infty} A_{j}\right\} \longrightarrow L
$$

by $H_{0}\left|L \times\{0\}=\bar{h}, H_{0}\right| L \times\{1\}=h^{\prime}$ and $H_{0}(v, t)=\varepsilon_{v}(t)$. Given $v \in A_{j}$ and $w \in A_{j+1}$, it is obvious that the loop $H_{0}(\overline{v w} \times\{0,1\} \cup\{v, w\} \times I)$ is null-homotopic in $L^{\prime}-L_{n_{j}}^{\prime}$. So $H_{0}$ extends to a p-homotopy $H$ between $\bar{h}$ and $h^{\prime}$, which is easily checked to be a p-homotopy of pairs.

COROLlaRY 2.5. $h:\left(\mathscr{F}(G), \mathscr{F}\left(G^{\mathrm{ns}}\right)\right) \rightarrow\left(\mathscr{F}\left(G^{\prime}\right), \mathscr{F}\left(G^{\prime n s}\right)\right)$ is a homeomorphism if and only if $h^{\prime}$ is a p-homotopy equivalence. 
LEMMA 2.6. Let $f: A \rightarrow X$ be a p-map with the proper homotopy extension property and $h: X \rightarrow Y$ a p-homotopy equivalence. Then $A \cup_{f} X$ is p-homotopically equivalent to $A \cup_{h f} X$.

Proof. It is straightforward, following with minor changes the proof in ordinary homotopy (see [7, I.3.10]).

THEOREM 2.7. Two graphs $G$ and $G^{\prime}$ have the same p-homotopy type if and only if their characteristic pairs are isomorphic.

Proof. By Proposition 2.2 we can consider these graphs canonical. If they are both $\infty$-stable the result follows from Proposition 2.4. Assume that they have nonstable ends and let $h:\left(\mathscr{F}(G), \mathscr{F}\left(G^{\mathrm{ns}}\right)\right) \rightarrow\left(\mathscr{F}\left(G^{\prime}\right), \mathscr{F}\left(G^{\prime \text { ns }}\right)\right)$ be a homeomorphism and $T$ and $T^{\prime}$ the trees of $G$ and $G^{\prime}$ respectively. By Corollary 2.5 there is a p-homotopy equivalence of pairs $h^{\prime}:\left(T, T_{0}\right) \rightarrow\left(T^{\prime}, T_{0}^{\prime}\right)$, where $T_{0}$ and $T_{0}^{\prime}$ are the corresponding non-stable subtrees. Actually, given any increasing sequence of compact subtrees $L_{1}^{\prime} \subset L_{2}^{\prime} \subset \ldots$ in $T^{\prime}$, we may find a sequence of compact subtrees $L_{1} \subset L_{2} \subset \ldots$ in $T$ such that if $A_{j}, B_{j}$ are the sets of first vertices of components of $T-L_{j}$ and $T^{\prime}-L_{j}^{\prime}$ respectively, $h^{\prime}$ maps $A_{j}$ into $B_{j}$ and all the vertices of $L_{j}-L_{j-1}$ into $B_{j-1}$ (see the proof of Proposition 2.4). Now by Lemma 2.6, if we attach one copy of $S^{1}$ to $h^{\prime}(v)$ for each copy of $S^{1}$ attached at $v$ we obtain a new graph $G^{*}$ in the p-homotopy class of $G$ with tree $T^{\prime}$. We finally apply Proposition 2.4 to reduce $G^{*}$ and $G^{\prime}$ to the same graph up to $\mathrm{p}$-homotopy equivalence.

ACKNOWLEDGEMENTS. This work was partially supported by the projects CAICYT 0812-84 and 'Consolidación de grupos de Trabajo'-PAICYT (Andalucia).

\section{References}

1. E. DomingueZ and L. J. Hernández, 'Remarks about proper ends', Rev. Roumaine Math. Pures Appl., to appear.

2. F. T. FARREL, L. R. TAYLOR and J. B. WAGONER, 'The Whitehead Theorem in proper category', Compositio Math. 27 (1973) 1-23.

3. H. Freudenthal, 'Uber die Enden topologischer Raume and Gruppen', Math. Zeit. 33 (1931) 692-713.

4. W. MASSEY, Algebraic topology. An introduction, Graduate Texts in Math. 56 (Springer, Berlin, 1977).

5. M. MihaliK, 'Semistability at infinity and the end of a group extension', Trans. Amer. Math. Soc. 277 (1983) 307-321.

6. I. Richards, 'On the classification of noncompact surfaces', Trans. Amer. Math. Soc. 106 (1963) 259-269.

7. V. Rohlin and D. Fuchs, Premier course de topologie (Mir Publishers, Moscow, 198I).

\author{
R. Ayala, A. Marquez and A. Quintero \\ Facultad de Matematicas \\ Universidad de Sevilla \\ 41080 Sevilla \\ Spain
}

\author{
E. Dominguez \\ Departamento de Matematicas \\ Facultad de Ciencias \\ Universidad de Zarazoga \\ 50009 Zarazoga \\ Spain
}

